\title{
USO DE PREGUNTAS COMO ESTRATEGIA CLAVE EN LA SALA DE CLASES: LA PIEZA QUE FALTA
}

\author{
THE USE OF QUESTIONS AS A CORE \\ CLASSROOM STRATEGY: THE MISSING KEY
}

\author{
María Francisca Valenzuela Hasenohr \\ Antonietta Ramaciotti Ferré
}

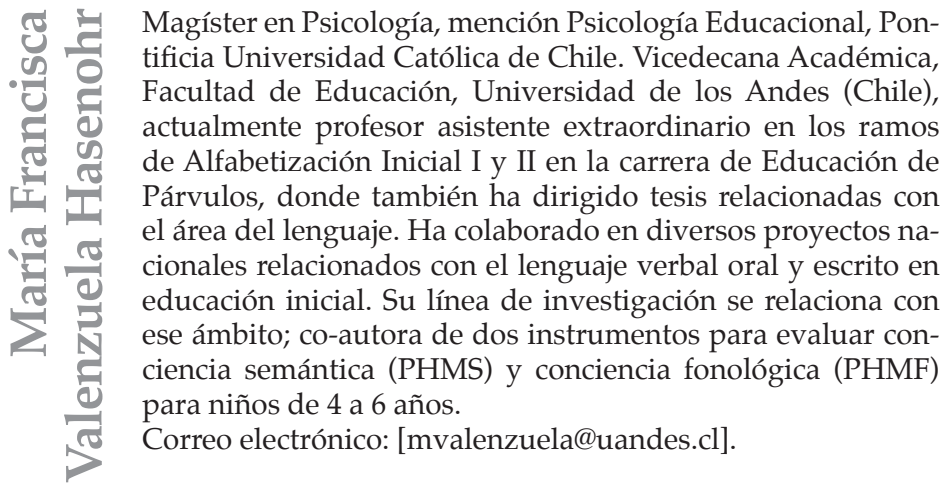


Magíster en Educación Especial y Diferencial, Pontificia Universidad Católica de Chile. Directora del Programa de Magíster en Psicopedagogía, Universidad de los Andes. Directora de la Escuela de Pedagogía Básica, Universidad de los Andes, en la categoría de profesor asistente ordinario. Ha dictado diversos cursos sobre estrategias y evaluación para la estimulación del lenguaje oral y escrito. Actualmente dicta cursos en el Magíster de Psicopedagogía relacionados con evaluación y diagnóstico, y dirige tesis relacionadas con el área del lenguaje. Ha participado en diversos proyectos nacionales, siendo el más importante el proyecto ELEA (Programa para la estimulación del lenguaje oral y escrito: un modelo de continuidad), creando varios juegos para el desarrollo metalingüístico, mismos que se utilizan en diversos centros escolares del país y fuera de Chile. Co-autora de la Prueba de segmentación lingüística (PSL), para niños de 5 a7 años de edad.

Correo electrónico: [aramaciotti@uandes.cl].

\section{RESUMEN}

Este artículo tiene por objetivo presentar el estado del arte en relación con el uso de preguntas en la sala de clases, como estrategia clave para el desarrollo del pensamiento del educando, en el ámbito de la educación preescolar. Para ello se revisaron publicaciones principalmente de habla inglesa, ya que existe una escasez de ellas en español. Se agrega una clasificación de la terminología empleada por distintos autores, referida a diversos tipos de preguntas y de ciertos factores que afectan su formulación. El uso de preguntas desafiantes en la sala de clases favorece el desarrollo de un pensamiento de buena calidad, extiende el vocabulario y mejora la comprensión. Se incluyen las principales características que deben considerar los docentes al formular preguntas, para utilizarlas como una herramienta de aprendizaje efectivo, y que favorezca el desarrollo de un pensamiento de buena calidad entre los alumnos. Se busca generar discusión en torno a su empleo como estrategia pedagógica central que favorezca mejores aprendizajes, en forma especial en los años preescolares.

Palabras clave: preguntas; estrategia pedagógica; aprendizaje; pensamiento de buena calidad. 


\section{ABSTRACT}

The aim of this article is to review the research about the use of questions in the classroom, as a key strategy for developing thinking, in the field of early childhood education. To this end, publications that support the importance of questions in this educational space were reviewed, especially from English-speaking countries, due to the lack of research in Spanish-speaking preschool contexts. Additionally, the terminology used by different authors referring to different types of questions has been classified along with factors that affect question formulation. The development of good quality thinking, the extension of vocabulary and improved comprehension are some of the benefits discussed. Finally, different characteristics to be considered by teachers when ask questions, so that they become an effective learning tool for students, are mentioned. This review seeks to generate discussion about the use of questions as a core pedagogical strategy that promotes significant learning, especially in early childhood.

Keywords: questions; pedagogical strategy; learning; good quality thinking.

\section{INTRODUCCIÓN}

El proceso educativo que surge al interior de la sala de clases se basa en la interacción y el diálogo pues, como espacio de aprendizaje que es, debe ser potenciado (Nystrand, Wu, Gamoran, Zeiser y Long, 2003). Cada sala debe crear un entorno en que los alumnos tengan la oportunidad de pensar (Langer, 2000) y utilizar el lenguaje para manifestar ese pensamiento; es indispensable repensar el contexto de la sala para que ello efectivamente suceda, en el entendido que el discurso desplegado al interior de la sala promueve logros en los estudiantes. Las investigaciones muestran que los alumnos desarrollarán distintos tipos de aprendizaje dependiendo del énfasis que el profesor manifieste (Athanases, 1998).

En los últimos años, uno de los focos de la discusión se centra en aspectos cognitivos y de procesamiento lingüístico (Apleebe, Langer, Nystrand y Gamoran, 2003). Por ello es interesante detenerse en lo que 
realiza el profesor al interior de la sala de clases en relación con estos dos ejes; colocar la mirada en este tema puede iluminar algunos cambios que se requieren para alcanzar una educación efectiva. La sala de clases es un escenario complejo, en donde la instrucción involucra una negociación permanente entre profesor, alumno, materia y tiempo disponible. El proceso social que surge al interior de ella crea un contexto en el que los alumnos desarrollan la cognición y los procesos lingüísticos, lo que lleva a centrar el foco en estas habilidades para mejorar las prácticas educativas (Apleebe et al., 2003) y así optimizar el aprendizaje de los estudiantes.

\section{RELACIÓN ENTRE EL USO DEL LENGUAJE Y EL DESARROLLO DEL PENSAMIENTO}

Los primeros intentos por explicar la relación entre lenguaje y pensamiento poseían un enfoque conductista. Bermeosolo (2012) señala que para estos psicólogos, el aprendizaje de la lengua se adquiría sobre la base de datos que provenían del ambiente y que no dependían del nivel de inteligencia individual. Es decir, para estos ambientalistas, el aprendizaje del lenguaje se producía por condicionamiento o simple imitación.

Estudios posteriores, realizados desde la psicología cognoscitiva, permiten afirmar que el condicionamiento y la imitación pueden jugar un papel en la adquisición del lenguaje, sin embargo no explican lo esencial del proceso.

La psicolingüística chomskiana ha entregado uno de los aportes más significativos en el cambio de paradigma, transitando desde el conductismo hasta los aportes realizados por la teoría cognoscitivista. Los psicolingüistas innatistas plantean que el sujeto trae un repertorio lingüístico genéticamente determinado el cual puede desenvolverse con la influencia del exterior, y que actuaría sólo como un medio obstaculizador o potenciador del mismo (Bermeosolo, 2012). Desde un enfoque naturalista del estudio del lenguaje, Chomsky (1996) señala que la facilidad con que el niño aprende y desarrolla su lenguaje, y el nivel de elaboración cognitiva, difícilmente podría atribuirse a condicionamiento 
o simple imitación. Las propiedades biológicas de la condición humana determinan lo que él llamaría «un órgano del lenguaje», es decir, un tipo particular de mecanismo o dispositivo que el individuo posee genéticamente y que le preparará para la adquisición del lenguaje.

Otros estudios ya anteriores habían intentado explicar la relación pensamiento y lenguaje, como los realizados por Edward Saphir y Benjamin Whorf, quienes plantearon la «hipótesis del determinismo lingüístico»; señalaron que la palabra no tan sólo expresa una forma de pensar: el lenguaje interactuaría con el pensamiento y el comportamiento, influenciándose mutuamente. Saphir-Whorf postularon dos hipótesis explicativas (De Bustos Guadaño, 2004), la primera llamada hipótesis fuerte, señala que la lengua de una persona monolingüe determina la forma en que conceptualiza la información y la guarda en la memoria, lo cual determina sólidamente el pensamiento del hablante. La segunda, llamada hipótesis débil, plantea que el hablante influencia la forma en que organiza la información y cómo la guarda en la memoria. En síntesis, señalaban que el lenguaje determinaba el pensamiento del sujeto. Esta hipótesis fue refutada por Chomsky en torno a la existencia de un órgano universal del lenguaje pues, de lo contrario, cada hablante sólo conceptualizaría de acuerdo a su idioma materno y no podría elaborar conceptos universales.

Otros aportes importantes fueron los realizados por Jean Piaget. En su hipótesis explicativa señala que el individuo interactúa con el ambiente para organizar el conocimiento a partir de la acción (Piaget, 1991), modificando así sus esquemas mentales. Para este psicólogo, la fuente de las operaciones mentales se encuentra en la acción, pues ella permite al individuo organizar la experiencia y no así el lenguaje. Es decir, el origen del pensamiento estaría en la función simbólica, siendo el lenguaje una forma más de ella. A través de la acción, el individuo modificaría las estructuras lógicas (Cárdenas, 2011) y el lenguaje cumpliría la función de representación, cuyo objetivo sería reflejar el pensamiento.

Una mirada diferente sobre esta relación constituyó el aporte de Vygotsky (1989), quien en sus postulados señala que el lenguaje no tan sólo tendría la misión de reflejar el pensamiento, sino más bien, la tarea de transformar y modificar la función semiótica (aquélla encargada de 
lo simbólico e imaginario). Cárdenas (2011) reflexiona acerca de ambos planteamientos y señala que, a diferencia de Piaget, para Vygotsky los procesos de interiorización psicológica no se modifican por ser un reflejo de lo que ocurre en la realidad, sino más bien son producto de la interacción entre las personas. El lenguaje sería entonces un mediador que regula la forma de conocer y de comportarse de los humanos, incluso permitiría actuar sobre sus propias operaciones lingüísticas.

Mercer (2000), rescata los aportes de Vygotsky, al afirmar que el lenguaje constituye una herramienta que permite cumplir simultáneamente diferentes funciones: (i) una herramienta cognitiva que los estudiantes utilizan para procesar el conocimiento; (ii) una herramienta sociocultural que permite compartir el conocimiento; (iii) y una herramienta pedagógica que posibilita a una persona ser guía de otra. En este contexto, el autor señala que enseñar a pensar es, esencialmente, involucrar a los estudiantes en una práctica social que internaliza el lenguaje como una herramienta de pensamiento.

En relación con lo anterior, Dickinson y Caswell (2007) afirman que, en los primeros años, la naturaleza de la interacción profesor-alumno impacta el desarrollo del lenguaje del niño y su aprendizaje, pues el qué dice el docente y cómo lo dice tiene consecuencias en el educando. De hecho, Dickinson (2001a, 2001b) muestra efectos significativos del discurso del profesor, referidos a prácticas educativas desafiantes cognitivamente, en la frecuencia y extensión de la conversación, en el lenguaje de los alumnos. Pareciera que más importante que el «qué» enseñar es el «cómo» enseñarlo (Taylor, Pearson, Peterson y Rodríguez, 2003). Por esta razón, según Knapp (1995), los profesores efectivos someten a sus estudiantes a tareas de mayor demanda cognitiva, que implican habilidades tales como inferir, hipotetizar y evaluar, entre otras, lo cual mejoría su entendimiento, en relación con lo que deben hacer, el por qué y para qué de ello.

Respecto de cuál es el tipo de pensamiento que el lenguaje debiera fomentar dentro de la sala de clases, Beas, Santa Cruz, Thompsen y Utreras (2000) señalan que un pensamiento de buena calidad debe poseer tres características fundamentales: a) ser crítico, capaz de sustentar sus creencias, para lo cual el sujeto debe reelaborar la información; b) creativo, que permita ajustarse a situaciones nuevas y crear 
alternativas originales para solucionar una situación; y c) metacognitivo, capacitado para reflexionar sobre sus propios procesos.

Santiuste (2001) agrega que el pensamiento crítico es en sí, reflexivo y metacognitivo, característica que contribuye a que la persona pueda conocer su propio sistema cognitivo, es decir cuáles son sus conocimientos, motivaciones, estrategias y sentimientos.

Por otro lado, Marciales (2003) sostiene que cuando se habla de pensamiento crítico se alude a una serie procesos que lo integran. Es así como el contexto, las estrategias y las motivaciones deben ser consideradas. El contexto permite al individuo responder de manera acorde a la situación. Por otra parte, el individuo pondrá en juego todos los procedimientos de que dispone para enfrentar dicha situación; por último, y no menos importante, será la motivación que el individuo presente frente a dicha tarea, el nexo afectivo que «gatille» la curiosidad frente al nuevo conocimiento.

Este tipo de pensamiento permite que el individuo recurra a un potencial cognitivo que generalmente no ocupa. Feuerstein (1991) plantea que en la mayoría de los individuos existe una tendencia a realizar un análisis superficial de las situaciones, considerando generalmente un punto de vista, por lo tanto los argumentos suelen ser pobres, con escaso aporte de evidencias para fundamentar los juicios. Una de las problemáticas de la educación actual es revertir el predominio de un pensamiento superficial en pos de alcanzar el desarrollo de habilidades cognitivas más complejas que permitan a la persona alcanzar un pensamiento de calidad.

El pensamiento de buena calidad involucra el razonar y requiere de conocimiento basado en los hechos para ir más allá de ellos; el conocimiento es empleado para inferir. Este razonar es especialmente importante para la comprensión auditiva y lectora, por lo que es esencial desarrollarlo tempranamente (Duke y Carlisle, 2011). El desafiar al niño a través del lenguaje para alcanzar este tipo de razonamiento surge como una herramienta pedagógica indispensable para favorecerlo.

El lenguaje sería el vehículo que permitiría el desarrollo de estas habilidades cognitivas. Bermeosolo (2012) afirma que el individuo intenta 
reordenar la realidad: compara, agrupa, clasifica y organiza para responder a diversos cuestionamientos. Plantea que desde allí se posibilita el desarrollo del pensamiento crítico, esencial para favorecer una verdadera comprensión por parte de los alumnos versus una visión simplificadora de la información relacionada con los acontecimientos.

El habla actuaría así como un mediador del aprendizaje, pues se aprende en y a través del lenguaje (Vygotsky, 1989). De acuerdo a este autor, los patrones de habla surgidos al interior de la sala, modelan el tipo de aprendizaje logrado. Para promover niveles altos de pensamiento, deben implicar que el sujeto haga algo más que sólo recitar aquello que ha escuchado. De esta forma, se proponen prácticas pedagógicas desde una conceptualización que considere al individuo como un reelaborador de la realidad, potenciando un pensamiento de buena calidad que supone, necesariamente, estrategias que estimulen el procesamiento cognitivo, que inviten a la reflexión sobre el propio pensamiento y que no esperen una respuesta única como solución a un problema o una situación.

\section{LA PREGUNTA COMO ESTRATEGIA LINGÜÍSTICA QUE PROMUEVE EL PENSAMIENTO}

El facilitar oportunidades a los alumnos para reflexionar críticamente a través del lenguaje es una cuestión central en el quehacer pedagógico; las preguntas juegan un rol fundamental en esta reflexión, ya que ellas constituyen aproximadamente un tercio de las expresiones empleadas por los educadores en las salas de educación inicial (De Rivera, Giralometto, Greenberg y Weitzman, 2005). De acuerdo a Langer (2000), los profesores deben asumir que preguntar es parte de la naturaleza del proceso de entender un material nuevo. Más que ser ellas un indicador de falla en el proceso de enseñanza/aprendizaje, son un excelente punto de partida, esencial para iniciar la discusión.

Sin embargo, los profesores tienden a pensar que si no surgen preguntas dentro de la sala es porque todo está claro para los alumnos. La realidad es que el tipo de preguntas que el profesor elabora limita el tipo de procesamiento cognitivo realizado por los estudiantes y, 
por ende, el tipo y la complejidad de las respuestas que ellos proporcionarán. De hecho, Ernst (1994) observó que si el profesor formula preguntas de exposición (que piden información recién recibida), las respuestas de los alumnos son cortas, basadas en la repetición y con poca elaboración personal. Claramente, la participación del alumno y el lenguaje que éste utiliza es facilitado o restringido, dependiendo del tipo de preguntas realizadas por el educador, por la retroalimentación entregada, la extensión y organización de turnos de conversación y quién detenta el control del tema del cual se discute. Por esta razón, los profesores efectivos son aquellos que formulan preguntas de alto nivel, promoviendo procesos cognitivos superiores en sus estudiantes (Langer, 2001).

De esta forma, el rol del profesor consiste en impulsar el intelecto del alumno hacia nuevas ideas, profundizando el conocimiento. Para ello debe saber qué y cuándo preguntar, formular cuestionamientos amplios, en donde la respuesta correcta o errónea no sea claramente identificable. Se asume que la interacción profesor-alumno es frecuentemente iniciada por el docente, por lo que se requiere pensar con cuidado qué tipo de preguntas se realizarán al educando: él debe generar el tipo de pensamiento deseado, induciendo al alumno hacia niveles cada vez más complejos (Flanders, 1973). Danis, Bernard y Leproux (2000) revelan que el nivel de abstracción en una conversación depende del nivel que expresa quien pregunta, mostrando que los adultos elevan dicho nivel con mayor frecuencia que los niños y crean, así, una zona de desarrollo próximo (Arancibia, Herrera y Strasser, 2011). Los adultos deben ayudar al niño a desplegar habilidades de representación, permitiéndoles alcanzar niveles cada vez más complejos de abstracción.

Se necesita valorar el empleo de preguntas en diferentes contextos instruccionales como estrategia potente de desarrollo del pensamiento, a través del lenguaje. La importancia radica en formular preguntas abiertas al interior de la sala; es decir, aquellas que no admiten una respuesta única o su respuesta no es conocida por el profesor. Por ello, el alumno no tiene miedo de aventurarse en contestar, no siente temor de ser enjuiciado por aquello que exprese (Johnson, Ivey y Faulkner, 2011). Concuerdan con esta idea Wittmer y Honing (1991) quienes afirman 
que es indispensable formular este tipo de preguntas a los alumnos, pues ellas facilitan el desafío cognitivo a través de la respuesta.

Las preguntas de los adultos aumentan la participación verbal de los niños en sus actividades (Justice, Weber, Ezell y Bakeman, 2002). La bibliografía revisada concuerda en que se requiere del trabajo con preguntas que generen niveles más altos de razonamiento - como es el caso del uso de preguntas inferenciales (McKeown y Beck, 2003; Van Kleeck, 2003)—, pues el niño preescolar no se desplazará del nivel literal al inferencial, a menos que el adulto lo invite a ello (Danis, Bernard y Leproux , 2000).

\section{OTROS BENEFICIOS DEL USO DE PREGUNTAS COMO ESTRATEGIA DE APRENDIZAJE}

El empleo de preguntas es una herramienta pedagógica que favorece no sólo el desarrollo de las habilidades de pensamiento en general de los estudiantes; también la literatura revisada destaca otros beneficios asociados al proceso de alfabetización, como el desarrollo de vocabulario y la mejora de los procesos de comprensión en forma específica.

\section{Vinculación del uso de preguntas con desarrollo vocabulario.-}

Lonigan y Whitehurts (1998) afirman que el empleo de preguntas durante la lectura de cuentos, es una herramienta poderosa que colabora al desarrollo del vocabulario en el niño. Ewers y Brownson (1999) demuestran que el vocabulario receptivo es mayor en niños a quienes se enseñan palabras nuevas a través de preguntas (por ejemplo: «iqué es una posada?»), en comparación a cuando se hace a través de la designación (por ejemplo: «esto es una posada»). Wasik y Bond (2001) confirman esta idea: el uso de preguntas literales e inferenciales, durante la lectura de cuentos, favorece el desarrollo de vocabulario receptivo y expresivo, como también de habilidades de lenguaje literal e inferencial. 
Dickinson y Smith (1994), muestran la relación entre la exposición del niño al lenguaje inferencial en la discusión de lecturas (análisis, predicción), y las habilidades de vocabulario receptivo en niños de kínder. Agregan que la aplicación excesiva de preguntas rápidas, es decir, aquéllas en que el adulto sabe la respuesta - tales como: ¿qué?, ¿quién?, ¿cuándo?, ¿cómo?, en relación con una lectura-, excluye otro tipo de interrogantes más desafiantes. Por ello sugieren combinar ambos tipos de preguntas.

Confirman esta idea Paratore, Cassano y Schickedanz (2011) al indicar que los estilos de conversaciones adulto-niño —específicamente el formular al niño preguntas cognitivamente desafiantes, que inviten a establecer relaciones lógicas entre objetos, eventos, conceptos o conclusiones-, ayuda al desarrollo del vocabulario y de otros aspectos relacionados con la alfabetización. Dickinson y Smith (1994) señalan, igualmente, que la exposición de los niños a lenguaje inferencial -incluidas las preguntas de este tipo- se relaciona con un incremento superior en el vocabulario receptivo de niños de kínder. El incluir ambos tipos de preguntas (literales e inferenciales) proporciona mejores resultados en el vocabulario receptivo y expresivo (Wasik y Bond, 2001).

Justice (2002) afirma lo contrario: el enseñar vocabulario, etiquetando palabras, produce mejores resultados que a través de preguntas; pero este hallazgo se refiere sólo a vocabulario receptivo, pues ocurre lo contrario en relación con el vocabulario expresivo. La autora interpreta estos resultados al indicar que esa forma de enseñar palabras nuevas produce mejores resultados sólo a corto plazo, en contraste con el uso de preguntas como estrategia de enseñanza de vocabulario, misma que permite al niño un aprendizaje gradual y mejor desempeño tanto expresivo como receptivo.

Las investigaciones, entonces, afirman que el vocabulario del niño mejora cuando los adultos le formulan preguntas, ya que ello impulsa el empleo de nuevas palabras aprendidas. 


\section{Vinculación del uso de preguntas con la comprensión}

Según Duke y Carlisle (2011) es fundamental entender la naturaleza del proceso comprensivo para, de esa forma, ofrecer las mejores estrategias que favorezcan su desarrollo en el estudiante. Estos autores definen comprensión como el acto de construir significado con un texto oral o escrito, enfatizando que se trata de un proceso constructivo. El Reading Study Group (2002, en Duke y Carisle, 2011) señala que en este proceso se involucran diversos factores, pudiendo agruparse en aquéllos relacionados con el texto (su lenguaje, contenido, estructura, propósito y rasgos); con quien escucha o lee (conocimiento previo, propósitos, estrategias y habilidades), y con el contexto en que surge la comunicación.

Las preguntas pueden transformar precisamente ese contexto educativo en un escenario que favorezca el proceso comprensivo del alumno. Ya que el producto de la comprensión —el significado- no es estable, formular preguntas cambia la interacción del sujeto con lo que escucha $-\mathrm{o}$ con lo que lee-, enriqueciendo la comprensión (Wilkinson y Hye Son, 2011).

Por ejemplo, estudios de Nystrand (1997), y Nystrand y Gamoran (1991), muestran que ciertos rasgos de discusión grupal generada en clases, se relacionan positivamente con la comprensión de los alumnos. Cuando los profesores formulan preguntas auténticas (es decir, aquellas que no tienen una respuesta única y específica, y las preguntas consideran las respuestas previamente proporcionadas por los alumnos), los niños comprenden mejor. Applebee et al. (2003) confirman en su estudio que el empleo de este tipo de preguntas, en contextos académicamente desafiantes, se relaciona positivamente con la comprensión. De acuerdo a Cain y Oakhill (1999), las preguntas que favorecen el razonamiento analógico y causal se vinculan, posteriormente, con habilidades comprensivas.

La comprensión es un proceso dinámico y la instrucción puede afectarla (Duke y Pearson, 2002). Por ello, Duke y Carlisle (2011) sostienen que es necesario desarrollar ciertos hábitos en la forma de pensar en los 
primeros años, por su vinculación con la comprensión. La habilidad de inferir aparece como un factor de predicción; emplear preguntas que ayuden al niño a desarrollarla, aflora como algo esencial. Lawrence y Snow (2011) afirman que la práctica pedagógica de preguntar al autor en forma oral se asocia con mejores niveles de comprensión posterior. Ellos señalan que el diálogo entre profesor y alumno - que incluye preguntas inferenciales, predictivas e interpretativas- favorece el traspaso gradual de la responsabilidad desde el profesor al alumno en el aprendizaje, contribuyendo de esa forma a mejorar la comprensión. Los mismos autores afirman que el uso de preguntas abiertas, que requieren de un procesamiento de la información más profundo, se relaciona con mejores niveles comprensivos. Confirman esta idea Beck y McKeown (2006), quienes señalan que las preguntas deben ser cuidadosamente secuenciadas para ayudar a los estudiantes a comprender los elementos de la lectura, por lo que deben formularse de acuerdo a cada una de ellas y no en forma general. Sólo de esa manera el alumno relacionará la información escuchada o leída, favoreciendo el proceso comprensivo.

La habilidad de inferir en los años preescolares es predictiva de la comprensión lectora posterior; las raíces de este tipo de pensamiento se establecen muy temprano y es posible desarrollarla a través del buen empleo de preguntas. El problema está en que se observa una falta de intencionalidad de trabajo en el lenguaje en las salas preescolares, enfatizándose éste cuando los estudiantes ya leen fluidamente (Duke y Carlisle, 2011).

\section{TIPO DE PREGUNTAS EMPLEADAS EN LA SALA DE CLASES}

Más allá de todos los beneficios asociados al empleo de preguntas en la sala, no existe coincidencia en el uso de una terminología común que ayude al docente a valerse de esta información para mejorar sus prácticas pedagógicas. Por esta razón, a continuación se ofrece un resumen de los principales tipos de preguntas que aparecen en la literatura revisada. 
Boyd y Rubin (2002) distinguen las preguntas formuladas en explícitas (se espera como respuesta que el alumno repita información recién recibida, siendo ellas un recurso muy empleado para evaluar contenidos) y preguntas auténticas (piden al alumno su propia evaluación e interpretación del contenido). Los autores afirman que son éstas las que infieren, predicen, hipotetizan y evalúan, por lo que deberían emplearse con mayor frecuencia en la sala de clases. Los autores sostienen que las preguntas sólo referidas a los eventos de un texto, anulan el diálogo en torno a él mismo, pues al dar la única respuesta, éste finaliza. Afirman que deben crearse deliberadamente oportunidades en que las preguntas cumplan un rol central, que posibiliten al alumno iniciar temas de discusión y elaborar respuestas propias. En vez de clases tradicionales en que el profesor habla y los alumnos escuchan, se sugiere incorporar preguntas contingentes en la sala de clases, que permitan al educador escuchar al alumno y favorezcan alcanzar un nivel en que él mismo se cuestione el conocimiento.

Nystrand et al. (2003) clasifican las preguntas en preguntas no auténticas (también las llaman de «bajo orden») que no demandan mayor elaboración y tienen sólo una posible respuesta; y preguntas auténticas (o «de alto orden»), que involucran un mayor procesamiento cognitivo en la elaboración de la respuesta. Éstas últimas no pueden ser contestadas únicamente considerando el conocimiento anterior: requieren de un procesamiento de la información más complejo (generalizar, analizar, especular), no tienen una única posibilidad como respuesta e implican considerar la respuesta del niño para volver a preguntar.

Wasik (2010) se refiere a preguntas cerradas, aquellas que se responden sólo con un sí o un no; y preguntas abiertas, que necesitan más de una palabra como respuesta y promueven el uso amplio del lenguaje oral. La autora creó un programa (ExCELL) que incluye el empleo de preguntas abiertas, para favorecer el lenguaje en general de manera significativa, y en forma específica el vocabulario, en niños asistentes a pre-kínder y kínder. En él, incluye asesoramiento y acompañamiento a profesores, para modificar sus conductas al interior de la sala e impactar positivamente en el desarrollo del lenguaje de los alumnos. Entre los cambios sugeridos aparece el entrenamiento para impedir que una conversación se termine con una sola 
respuesta a las preguntas efectuadas, rescatando así la importancia de tomar la respuesta del niño para «gatillar» nuevas preguntas que desafíen al estudiante; ello implica escuchar activamente la respuesta y en forma explícita solicitar más información, generando niveles de procesamiento cada vez más complejos.

Zucker, Justice, Piasta y Kaderavek (2010) proponen una clasificación dependiendo del nivel cognitivo que se demande, basándose en que las habilidades lingüísticas emergen en el contexto de la interacción con otros (Chapman, 2000). Es así como distinguen entre el lenguaje literal (aquél en que el niño debe discutir, describir o responder a información recibida) y el lenguaje inferencial (las habilidades lingüísticas se emplean para abstraer, analizar, hipotetizar o reflexionar acerca de la información). Este último conlleva un mayor desafío cognitivo, un nivel de representación más complejo, pues las preguntas literales requieren menor procesamiento cognitivo que las inferenciales, por el tipo de lenguaje empleado. Las habilidades lingüísticas inferenciales son importantes para la comprensión lectora, ya que los autores de los textos dejan ideas en forma implícita, por lo que la información que no está proporcionada directamente por el texto, requiere deducirse. De acuerdo a Snow (1991), el facilitar al niño oportunidades de desarrollar el lenguaje inferencial, previene dificultades de comprensión lectora; por ello la importancia de su trabajo en los primeros años. El incorporar este tipo de preguntas durante la lectura compartida de cuentos, por ejemplo, es una buena oportunidad, cuidando de no utilizar en forma excesiva preguntas de tipo literal, o «test questions» (aquéllas en que el adulto sabe la respuesta), pues en ellas se tiende a contestar en forma mecánica, sin mediar el pensamiento.

Peterson y Taylor (2012) se refieren a preguntas de bajo nivel, dirigidas a un único tipo de respuesta de escaso procesamiento cognitivo; y preguntas de alto nivel, aquellas que requieren pensar en un nivel más profundo para elaborar la respuesta e incluyen muchas palabras en su elaboración. Las segundas enfatizan la interpretación de los personajes y ayudan a los estudiantes a hacer conexiones entre el texto y sus propias vidas. Facilitan también el que los niños compartan distintos puntos de vista y potencian el que sean ellos mismos quienes luego creen sus propias preguntas de alto nivel, favoreciendo la discusión 
grupal. Los autores indican que en un principio no es fácil para los alumnos, pero con la ayuda del docente, son ellos mismos quienes luego formulan preguntas desafiantes que provocan al pensamiento.

A partir de la clasificación antes descrita es posible señalar que más allá de las diferencias terminológicas, todos los autores concuerdan en que las preguntas a emplear al interior de la sala de clases pueden clasificarse en dos grandes grupos, dependiendo del nivel de procesamiento cognitivo que éstas demanden. Por esta razón, se ha elaborado la siguiente tabla que ilustra que, independientemente de la nomenclatura usada por distintos autores, la literatura revisada distingue distintos tipos de preguntas según el procesamiento de la información que se requiera para responderlas, y que es necesario considerarlo al momento de formularlas en la sala de clases, para favorecer el procesamiento cognitivo del niño.

\begin{tabular}{|l|l|l|l|l|}
\hline $\begin{array}{c}\text { Nivel } \\
\text { cognitivo }\end{array}$ & $\begin{array}{l}\text { Terminología } \\
\text { original }\end{array}$ & \multicolumn{1}{|c|}{ Características } & \multicolumn{1}{|c|}{ Ejemplos* } & \multicolumn{1}{c|}{ Autores } \\
\hline \multirow{4}{*}{ Bajo } & Exposición & $\begin{array}{l}\text { Repetición de información } \\
\text { recién recibida. }\end{array}$ & $\begin{array}{l}\text { ¿Qué lugares } \\
\text { visitó...? }\end{array}$ & $\begin{array}{l}\text { Boyd y Rubin } \\
\text { (2002). }\end{array}$ \\
\cline { 2 - 5 } & $\begin{array}{l}\text { No } \\
\text { auténticas }\end{array}$ & $\begin{array}{l}\text { Revisan que el alumno } \\
\text { sepa «lo que debe saber». }\end{array}$ & $\begin{array}{l}\text { ¿Cuáles eran los } \\
\text { nombres de los } \\
\text { personajes que } \\
\text { aparecían en el } \\
\text { cuento? }\end{array}$ & $\begin{array}{l}\text { Nystrand, } \\
\text { Wu, } \\
\text { Gamoran, } \\
\text { Zeiser y } \\
\text { Long (2003). }\end{array}$ \\
\cline { 2 - 6 } & \multirow{2}{*}{ Cerradas } & $\begin{array}{l}\text { Requieren de un «sí» 0 } \\
\text { «no" por respuesta. }\end{array}$ & $\begin{array}{l}\text { ¿Les gustó el } \\
\text { cuento? }\end{array}$ & $\begin{array}{l}\text { Wasik (2010) } \\
\text { Zucker. }\end{array}$ \\
\cline { 2 - 5 } & Literales & $\begin{array}{l}\text { Implican responder } \\
\text { describiendo información } \\
\text { que se ha recibido. }\end{array}$ & ¿Dónde vivía...? & $\begin{array}{l}\text { Justice, } \\
\text { Piasta y } \\
\text { Kaderavek } \\
\text { (2010). }\end{array}$ \\
\cline { 2 - 5 } & Bajo nivel & Sólo un tipo de respuesta. & $\begin{array}{l}\text { ¿Cómo se llamaba } \\
\text { el protagonista de la } \\
\text { historia? }\end{array}$ & $\begin{array}{l}\text { Peterson y } \\
\text { Taylor (2012). }\end{array}$ \\
\hline
\end{tabular}




\begin{tabular}{|c|c|c|c|c|}
\hline \multirow{4}{*}{ Alto } & Auténticas & $\begin{array}{l}\text { Interpretación y evaluación } \\
\text { de la información; implica } \\
\text { negociar significados. } \\
\text { No tienen una respuesta } \\
\text { única; exploran distintos } \\
\text { entendimientos. }\end{array}$ & $\begin{array}{l}\text { ¿Por qué piensas tú } \\
\text { que pasó eso? } \\
\text { ¿Por qué el autor } \\
\text { habrá escrito este } \\
\text { cuento? }\end{array}$ & $\begin{array}{l}\text { Boyd y Rubin } \\
\text { (2002). } \\
\\
\text { Nystrand, } \\
\text { Wu, } \\
\text { Gamoran, } \\
\text { Zeisery } \\
\text { Long } \\
\text { (2003). } \\
\end{array}$ \\
\hline & Abiertas & $\begin{array}{l}\text { Van más allá de la } \\
\text { información textual } \\
\text { explícita; requieren mayor } \\
\text { información que una } \\
\text { palabra como respuesta. }\end{array}$ & $\begin{array}{l}\text { ¿Qué piensas tú que } \\
\text { hará después...? }\end{array}$ & $\begin{array}{l}\text { Long (2003). } \\
\text { Wasik (2010). }\end{array}$ \\
\hline & Implícitas & $\begin{array}{l}\text { Requieren el uso de } \\
\text { habilidades lingüísticas } \\
\text { para abstraer o analizar } \\
\text { información }\end{array}$ & $\begin{array}{l}\text { ¿Cómo crees que se } \\
\text { sintió...? ¿Por qué? }\end{array}$ & $\begin{array}{l}\text { Zucker, } \\
\text { Justice, } \\
\text { Piasta y } \\
\text { Kaderavek } \\
\text { (2010). } \\
\end{array}$ \\
\hline & Alto nivel & $\begin{array}{l}\text { Requieren de un nivel de } \\
\text { pensamiento más profundo; } \\
\text { generan en el niño } \\
\text { preguntas de igual nivel. }\end{array}$ & $\begin{array}{l}\text { ¿Cómo sabes que } \\
\text { alguien es tu amigo? }\end{array}$ & $\begin{array}{l}\text { Peterson y } \\
\text { Taylor (2012). }\end{array}$ \\
\hline
\end{tabular}

* Ejemplos referidos a la lectura de un cuento, para niños de edad preescolar (4 a 6 años).

En resumen, los autores distinguen preguntas de dos tipos: unas requieren de mayor elaboración en la respuesta, involucran un lenguaje más complejo, niveles de procesamiento de la información recibida más altos y muchas veces no presentan una sola respuesta; en cambio, otras solicitan repetición de información recibida, poseen una sola respuesta e involucran escaso procesamiento cognitivo, siendo las primeras las que debieran potenciarse al interior de la sala de clases, para promover un pensamiento de buena calidad. Cuando se 
formulan preguntas de alto orden - como estrategia frecuente de enseñanza aprendizaje-, las respuestas a ellas también implican mayor nivel de procesamiento de la información y, a su vez, los alumnos empiezan a crear sus propias preguntas complejas, dirigidas a sus pares, para discutir en grupo. Generar preguntas de alto orden puede ser difícil para el estudiante, por lo que se requiere de ayuda, en un inicio, por parte del educador.

A partir de lo señalado es posible mencionar que para mejorar el aprendizaje de los estudiantes y que ellos desarrollen habilidades de pensamiento superior, es necesario que las preguntas que los profesores formulen al interior de la sala sean principalmente aquellas que demandan un alto nivel de procesamiento cognitivo. Por esta razón, la siguiente sección hace referencia a la evidencia empírica que muestra qué tan complejas y qué tan demandantes, cognitivamente, son las preguntas que generalmente se usan al interior de la sala.

\section{EVIDENCIA EMPÍRICA RESPECTO DEL USO DE PREGUNTAS DE ALTO NIVEL COGNITIVO}

A pesar de la importancia de la formulación de preguntas con altos niveles de demanda cognitiva para promover el desarrollo del pensamiento de los estudiantes, las investigaciones muestran que ello no ocurre a menudo en la sala de clases. De acuerdo a Cullen (2002), la práctica educativa otorga escasas oportunidades a los estudiantes para preguntar, nombrar temas de interés y negociar significados. Cuando el niño tiene la oportunidad de pensar acerca de preguntas de alto nivel, necesita considerar distintos puntos de vista (Peterson y Taylor, 2012). Ello conduce a procesar la información en un nivel de complejidad alto, respondiendo al mismo nivel y favoreciendo la generación propia de preguntas más complejas.

Los aportes de Beas (1994) coinciden con el planteamiento de Cullen (2002), quien sostiene que el sistema educativo en general se contenta con que el alumno verbalice algunos elementos referidos a un contenido determinado, se le pide información que exige en general mayor capacidad de memoria, descuidando otras tareas cognitivas. Afirma que: 
[...] no sólo se enseña en forma superficial, sino también se evalúa estableciendo estándares que pueden ser acordes a la enseñanza, pero que están por debajo del potencial del alumno (Beas, 1994, p. 25).

Esto no ocurre en escuelas efectivas (Hoffman, 1991). Taylor, Pearson, Clark y Walpole (1999) señalan que entre las características de los profesores de estas escuelas, está el que ellos formulan con mayor frecuencia preguntas de más alto nivel a sus alumnos. Pues al vincular, por ejemplo, preguntas más complejas con el texto trabajado, se impulsa en el niño el hacer conexiones con el conocimiento que ya se tiene, promueve el considerar elementos temáticos, interpretar motivaciones y acciones, favoreciendo de esa forma la comprensión. En contraste, señalan que cuando se realizan preguntas de bajo nivel, se pide al alumno únicamente información acerca de detalles, requiriendo una respuesta de sólo una o dos palabras. Ello se traduce en una comprensión mecánica, en que los alumnos informan verbalmente o en una hoja de trabajo la idea principal o secundaria, hecho u opinión. Este tipo de actividades requieren de un esfuerzo cognitivo menor que las señaladas en un inicio.

\section{OTROS FACTORES QUE AFECTAN \\ LA CALIDAD DE LAS PREGUNTAS}

El nivel de procesamiento cognitivo no es el único factor que determina la calidad de las preguntas formuladas por los profesores en la sala, sino que la literatura señala una multiplicidad de éstos. Por esta razón, en esta sección se revisarán los principales factores que se asocian a la calidad de éstas, a saber: edad de los estudiantes, tipo de texto utilizado y tema que se esté tratando; así como también la vinculación entre preguntas y respuestas.

\section{Tipos de preguntas y edad}

Si bien hay estudios que muestran que no existen diferencias en el uso de diferentes tipos de preguntas según la edad de los niños (Marinac, 2000), existen otros (De Rivera, et al., 2005) que afirman 
lo contrario, señalando que los educadores emplean más preguntas abiertas que cerradas con niños mayores que con los más pequeños. Estos autores mostraron en un estudio exploratorio que los profesores que trabajan con niños preescolares (4 a 6 años), formulan mayor cantidad de preguntas que quienes trabajan con niños menores (3 años). Asimismo, que los niños preescolares responden más y mejor a preguntas abiertas que los niños de menor edad, pero la gran cantidad de preguntas cerradas que realizan los profesores de niños pequeños en general (tres veces más que las abiertas) sugiere que no consideran las habilidades lingüísticas del niño al formularlas.

\section{Tipos de preguntas y texto}

El producto de la comprensión de un texto no es estable, y las preguntas que se realicen en torno a éste pueden cambiar la interacción entre el lector y el texto, generando diversos niveles de comprensión (Wilkinson y Son, 2001).

Pareciera que existe relación entre el tipo de texto que se esté trabajando con el grupo de niños y el tipo de preguntas que, a partir de ellos, se generen. Existen estudios que señalan que los adultos formulan preguntas más desafiantes cuando se trabajan géneros informativos en comparación con géneros narrativos (Torr y Clugston, 1999).

Apoyan esta idea Zucker et al. (2010), al mostrar que los rasgos del texto afectan el tipo de preguntas formuladas, las respuestas de los niños y el vocabulario desarrollado, en un estudio llevado a cabo con niños de cuatro años. Los autores indican que el uso de preguntas inferenciales surge con mayor frecuencia cuando se leen textos informativos, pues éstos incluyen conceptos más abstractos, si se comparan con preguntas formuladas al leer textos narrativos. También observaron asociación entre textos de nivel literal y preguntas del mismo tipo, sin encontrar asociaciones entre textos inferenciales y preguntas de este nivel.

Cuando se leen textos narrativos, la mayoría de las conversaciones se centran en temas literales, más que en inferenciales. Danis, Bernard 
y Leproux (2000) indican que las preguntas literales representan alrededor de un $76 \%$; en cambio, las inferenciales sólo un $23 \%$ de la conversación extra textual, en un estudio en que observaron niños de 32 meses (promedio), que asistían a guarderías infantiles y los adultos a cargo. Beck y McKeown (2006) sostienen que son las propias características de los textos las que generan diversos tipos de preguntas, pues el propósito y la estructura varían de acuerdo a ellos. En el caso de los textos narrativos, se requiere de la formulación de preguntas que favorezcan la comprensión, pues este tipo de textos no siempre se desarrollan en línea cronológica y utilizan recursos literarios para trasmitir implícitamente ideas, lo que dificulta la formulación. Por ello, debiera planificarse cuidadosamente el tipo de preguntas que se dirigirá al alumno, dependiendo del texto al cual se le está enfrentando.

\section{Tipos de preguntas y tema}

Las preguntas deben sincronizarse con los temas de interés del niño, pues de esa manera ofrecen oportunidades reales de aprendizaje. Burton y Habenicht (2004) señalan que deben formularse preguntas que ayuden al niño a investigar sobre diversos temas, conduciendo a la reflexión. Esto supone que el profesor planifique con anticipación las preguntas que realizará, seleccionando contenidos que los lleven a desarrollar los distintos niveles de procesamiento de la información.

A ello agregan De Rivera et al. (2005) que los niños preescolares responden más a preguntas de profundización de un tema que de iniciación de uno. Confirman esta idea Neuman y Roskos (2012) al señalar que cuando se centra la discusión en un tema previamente abordado, las preguntas son más desafiantes, pues permiten mayor profundización del tópico en cuestión. Por ello, los educadores que trabajan con niños de educación inicial deben formular preguntas abiertas y de continuación de tópico, que permitan complejizar el nivel de las preguntas, favoreciendo de esa manera el desarrollo del pensamiento. 


\section{Vinculación entre tipo de preguntas y respuesta}

Por su naturaleza, las preguntas, requieren obligatoriamente de una respuesta, pues invitan al otro a participar explícitamente. Por ello, el tipo de pregunta promueve cierto tipo de respuesta. Para que ésta se dé, el docente debe dar tiempo a los alumnos para responderla, brindando oportunidades de procesar la información, convirtiéndola en un real instrumento pedagógico (Zuleta, 2005).

Swain (2000) indica que la respuesta del niño requiere de un esfuerzo mental, lo cual implica que las preguntas inferenciales efectivamente empujan al niño a emplear un lenguaje cognitivamente desafiante, que incluye analizar e inferir. Zucker et al. (2010) observaron un alto nivel de asociación entre las preguntas inferenciales del profesor y las respuestas del mismo tipo por parte del niño, señalando que se sabe poco acerca de la frecuencia de uso de preguntas inferenciales durante las interacciones naturales que surgen en la sala de clases.

Peterson, Taylor, Burnham y Schock (2009) mencionan que con frecuencia el profesor elabora una pregunta, llama a un alumno a contestar y luego evalúa esa respuesta en términos de si ella es correcta (es decir, posee la información solicitada) o errada, sin provocar una interacción adulto-niño a partir de ella. Es decir, no considera la respuesta del niño para estimular un nivel de pensamiento más complejo. De Rivera et al. (2005) afirman que los educadores repiten rápidamente las preguntas, sin detenerse en saber si el niño entendió realmente la pregunta o en modificar la forma o el contenido de la misma, de manera que dé pistas sobre la respuesta. Las respuestas de los niños son importantes de estudiar, pues revelan el nivel de su compresión ante tópicos determinados.

McKeown y Beck (2003) observaron que, durante la lectura de cuentos, las preguntas inferenciales generan niveles más altos de razonamiento en las respuestas. Zucker et al. (2010), concuerdan con ello al sostener que el nivel de abstracción de las preguntas de los profesores se relaciona con el nivel de procesamiento de las respuestas de los niños. Se necesita acomodar las preguntas a la respuesta elaborada por el niño, de manera que desafíen progresivamente su intelecto. 
Un aspecto necesario a considerar es que, muchas veces, las preguntas se formulan de manera mecánica, sin considera las respuestas del niño. De acuerdo a Wilkinson y Hye Son (2011), el riesgo de que las estrategias se vuelvan mecánicas, radica en que la interacción profesor-alumno se vuelve altamente estructurada, al punto que inhibe el aprendizaje y la autorregulación en el uso de las estrategias de comprensión. Ello ocurre en ocasiones cuando lo que se aplican son «preguntas tipo» (qué, dónde, cuándo, cómo), donde la atención del profesor está más en formularlas todas, que en la respuesta del niño. La evidencia teórica sugiere modificar la siguiente pregunta, según la respuesta obtenida.

Otro aspecto a cuidar es que, a partir de las preguntas formuladas, las respuestas de los estudiantes no se transformen en una colección de comentarios (McKeown, Beck y Blake, 2009), unos seguidos de otros. Por el contrario, lo que se busca es que las respuestas de unos generen en el resto del grupo niveles más profundos de procesamiento de información, y por lo mismo, preguntas más complejas.

Como síntesis de los rasgos más relevantes que deben tener las preguntas, de acuerdo a la literatura revisada, es posible destacar que deben formularse preguntas abiertas; utilizar diferentes tipos de textos de base; continuar con el tema del cual se está conversando (más que cambiar en forma continua de tópico) para alcanzar mayores niveles de profundidad, y considerar la respuesta del niño para volver a preguntar, generando así niveles más altos de productividad en las interacciones al interior de la sala de clases.

\section{DISCUSIÓN E IMPLICACIONES}

El empleo de preguntas en la sala de clases como herramienta pedagógica que arroje un pensamiento de buena calidad, favoreciendo el aprendizaje del alumno, muestra tener respaldo teórico (Johnson, Ivey y Faulkner, 2011; Lawrence y Snow, 2011; Zucker et al., 2010; Applebee et al., 2003; Paratore, Cassano y Schickedanz, 2001; Danis, Bernard y Leproux, 2000; Langer, 2000; Wittmer y Honing, 1991). 
Elder y Paul (2002) sostienen que preguntar es el arte de pensar, es ofrecer una oportunidad de apertura al conocimiento. La forma en que los profesores usen el lenguaje al interior de la sala de clases importa; se requiere que las interacciones que allí se generen contribuyan efectivamente al aprendizaje de los educandos.

Entre los rasgos que destaca la literatura, para una adecuada formulación de preguntas, se encuentran la alta frecuencia del empleo de preguntas abiertas, que no tengan una sola respuesta, que consideren la respuesta del alumno al contra preguntar, que se basen en una variedad de textos y que permitan compartir diversas perspectivas ante un tópico determinado. El alumno debe tener la oportunidad de escuchar y elaborar preguntas, de vivir ambientes educativos que desafíen su intelecto, que fuercen a negociar significados y que inviten a pensar. El conocer las características de las preguntas de calidad, puede ayudar a crear ambientes de aprendizaje que potencien el pensamiento, a través del lenguaje, utilizando estrategias efectivas de aprendizaje.

Se necesita reflexionar acerca de qué clase de cambios introducir en las salas, qué prácticas pedagógicas potenciar para mejorar el aprendizaje de los alumnos. Se invita a centrar el foco de la discusión en qué elementos de intervención deben considerar los educadores y por qué. Se adelanta que el tipo de interacción entre profesor y alumno, y en forma específica, el tipo de preguntas que se formulen, es un tema central. Se deben crear deliberadamente oportunidades en que las preguntas cumplan un rol protagonista en la sala de clases: iniciar temas de discusión que permitan a los alumnos crear sus propias respuestas y estimular a que ellos elaboren sus propias preguntas (Boyd y Rubin, 2006).

La literatura revisada no hace referencia en torno al momento de la rutina en que esta herramienta pedagógica es más utilizada, si ella está relacionada exclusivamente con ciertos momentos o si existe vinculación respecto de los años de experiencia docente y su empleo. Ellos, entre otros, podrían ser focos de interés en futuras investigaciones. La revisión presentada en este artículo da cuenta de que existe investigación internacional de habla inglesa al respecto, pero es escasa en español para el ámbito preescolar, por lo que considerarla puede ser un punto de interés. 
El uso de preguntas como estrategia eficaz de enseñanza puede tomar años de experiencia, requiere de una considerable cantidad de horas invertidas en la sala y la solución de conflictos referidos a creencias y prácticas educativas (Wilkinson y Hye Son, 2011). A pesar de que cada profesor posee su propio estilo de enseñanza, es preciso considerar la incorporación de esta estrategia de manera permanente, pues el estudiante se verá beneficiado de ello.

Por tanto, ésta es una invitación a los docentes a reflexionar acerca de cómo y cuándo formular preguntas a los alumnos, pues es ahí donde es posible enfatizar el desarrollo del pensamiento a través del lenguaje, avanzando en el pensamiento de buena calidad y contribuyendo, así, a alcanzar la tan ansiada educación de calidad que todos los niños merecen.

\section{REFERENCIAS}

Arancibia, V. y Álvarez, M.I. (1994). Características de los profesores efectivos en Chile y su impacto en el rendimiento escolar y autoconcepto académico. PSYKE, 3(2), pp. 131-143.

Arancibia, V.; Herrera, P. y Strasser, K. (2011). Manual de Psicología Educacional. Santiago: Ediciones Universidad Católica.

Applebee, Q.N.; Langer, J.A.; Nystrand, M. \& Gamoran, A. (2003). Discussion-based approches to developing understanding: Classroom instruction and student performance in middle and high school English. American Education Research Journal, 40(3), pp. 685-730.

Athanases, S.Z. (1998). Diverse learners, diverse texts: Exploring identity and difference through literary encounters. Journal of Literacy Research, 30(2), pp. 273-296.

Beas, J.; Santa Cruz, J.; Thompsen, P. y Utreras, S. (2000). Enseñar a pensar para aprender mejor. Santiago: Ediciones Universidad Católica. 
Beas, J. (1994). ¿Qué es el pensamiento de buena calidad? Pensamiento Educativo, 5, pp. 13-28.

Beck, I.L. \& Mckeown, M.G. (2006). Improving comprehension with questioning the author. A fresh and expanded view of a powerful approach. USA: Scholastic.

Bermeosolo, J. (2012). Psicología del lenguaje: una aproximación pedagógica. Santiago: Ediciones Universidad Católica.

Boyd, M. \& Rubin, D. (2002). Elaborated student talk in an elementary ESoL classroom. Research the Teaching of English, 36, pp. 495-530.

Burton, L. y Habenicht, D.J. (2004). Lograr que los alumnos piensen. Uso de preguntas de modo efectivo en la sala de clases. Recuperado de http: / / circle.adventist.org files/jae/sp/jae2004sp191705.pdf

Cain, K. \& Oakhill, J.V. (1999). Inference making ability and its relation to comprehension failure in young children. Reading and writing: An interdisciplinary Journal, 11, pp. 489-503.

Cárdenas, A. (2011). Piaget: lenguaje, conocimiento y educación. Revista Colombiana de Educación, 60, pp. 71-91.

Cullen, R. (2002). Supportive teacher talk: The importance of the f-move. ELT Journal, 56, pp. 117-127.

Chapman, R. (2000). Children's language learning: An interactionist perspective. Journal of Child Psychology and Psychiatry, 41, pp. 33-54.

Danis, A.; Bernard, J. \& Leproux, C. (2000). Shared picture-book Reading: A sequential analysis of adult-child verbal interactions. British Journal of Developmental Psychology, 18, pp. 369-388. 
De Rivera, C.; Giralometto, L.; Greenberg, J. \& Wietzman, E. (2005). Children's responses to educator's questions in day care group play. American Journal Speech-Language Pathology, 14, pp. 14-26.

De Bustos Guadaño, E. (2004). Lenguaje, comunicación y cognición: temas básicos. Madrid: UNED.

Dickinson, D.K. (2001a). Book reading in preschool classrooms: Is recommended practice common? in D.K. Dikinson \& P.O. Tabors (Eds.). Beginning literacy with language: Young children learning al home and school (pp. 175-203). Baltimore: MD. Brookes Publising.

Dickinson, D.K. (20001b). Large-group and free-play times: Conversational settings supporting language and literacy development. In D.K. Dickinson \& P.O. Tabors (Eds.). Beginning literacy with language: Young children learning at home and school (pp. 223-255). Baltimore: MD Brookes Publishing.

Dickinson, D.K. \& Smith, M.W. (1994). Long-term vocabulary effects of preschool teacher's book Reading on low-income children's vocabulary and story comprehension, Reading Research Quarterly. 29, pp. 104-122.

Dickinson, D. \& Caswell, L. (2007). Building support for language and early literacy in preschool classrooms through in-service professional development: Effects of the Literacy Environment Enrichment Program (LEEP). Early Childhood Research Quarterly, 22, pp. 243-260.

Duke, N. \& Carlisle, J. (2011). The Development of Comprehension. In M.L. Kamil; P.D. Pearson; E. Moje \& P. Afflerbach (Eds.). Handbook of Reading Research, IV (pp. 199-228). New York: Routledge. 
Duke, N.K. \& Pearson, P.D. (2002). Effective practices for developing reading comprehension. In A.E. Farstrup \& S.J. Samuels (Eds.). What research has to say about reading instruction? (pp. 205-242). Newark DE: International Reading Association.

Elder, L. y Richard P. (2002). El arte de formular preguntas esenciales. Basado en conceptos de pensamientoo crítico y principios socráticos. Fundación para pensamiento crítico, pp. 1-39.

Ernst, G. (1994). Talking circles: Conversation and negotiation in the ESL classroom. TESOL Quarterly, 28, pp. 293-322.

Ewers, C.A. \& Brownson, S.M. (1999). Kindergarteners' vocabulary acquisition as a function of active vs passive storybook reading, prior vocabulary, and working memory. Reading Psychology, 20, pp. 11-20.

Feuerstein, R.; Klein, P. \& Tannenbaum, A. (1991). Mediated learning Experience (MLE): Theorical psychosocial and learning implications. England: Freund Publishing House Ltd.

Flanders, N.A. (1973). Basic teaching skills derived from a model of speaking and listening. Journal of Teacher Education, 24(1), pp. 24-37.

González., J.; Preiss, D. y San Martín, E. (2008). Evaluando el discurso docente: desarrollo de un modelo de Rasch a partir de la evidencia audiovisual de profesores chilenos de primer ciclo de educación básica en el área de lenguaje. Revista Iberoamericana de Evaluación Educativa, 1(2), pp. 138-147.

Hoffman, J.V. (1991). Teacher and School Effects in Learning to Read. In R. Barr; M.L. Kamil; P.B. Mosenthal, \& P.D. Pearson (Eds.). Handbook of Reading Research, 11 (pp. 911-950). New York: Longman.

Johnson, P.; Ivey, G. \& Faulkner, A. (2011). Talking in class. Remembering what in important about classroom talk. The Reading Teacher, 65(4), pp. 232-237. 
Justice, L.M. (2002). Word exposure conditions and preschooler's novel Word learning during shared storybook Reading. Reading Psychology, 23, pp. 87-106.

Justice, L.M.; Weber, S.E.; Ezell, H. K. \& Bakerman, R. (2002). A sequential analysis of children's responsiveness to parental print references during shared book-reading interactions. American Journal of Speech-Language Pathology, 11, pp. 30-40.

Knapp, M.S.; Adelman, N.E.; Maerder, C.; McCollum, H.; Needels, M.C.; Padilla, C.; Shields, P.M.; Turnbull, B.J. \& Zucker, A.A. (1995). Teaching for meaning in high-poverty classrooms. New York: Teachers College Press.

Langer, J.A. (2000). Excellence in English in middle and high school: How teachers' professional lives support student achievement. American Educational Research Journal, 37(2), pp. 397-439.

Langer, J.A. (2001). Beating the odds: Teaching middle and high school students to read and write well. American Research Journal, 38, pp. 837-880.

Larrain, A.; Howe, CH. y Cerda, J. (2014). Argumentación en enseñanza en clase completa y aprendizaje de ciencias. PSYKHE, 23(2), pp. 1-15.

Lawrence, J. \& Snow, C. (2001). Oral discourse and reading. In M.L. Kamil; P.D. Pearson; E. Moje \& P. Afflerbach (Eds.). Handbook of Reading Research, IV (pp. 320-337). New York: Routledge.

Leyva, D.; Barata, M.; Snow, C.; Weiland, CH.; Yoshikawa, H.; Treviño, E. \& Rolla, A. (2015). Teacher-Child Interactions in Chile and Their Associations With Prekindergarten Outcomes. Child Development, 00(0), pp. 1-19.

Lonigan, C.J. \& Whitehurts, G.J. (1998). Relative efficacy of parent and teacher involvement in a shared-reading intervention for preschool children from low-income backgrounds. Early Childhood Research Quarterly, 13, pp. 263-290. 
Marciales, G. (2003). Pensamiento crítico: diferencias en estudiantes universitarios en el tipo de creencias, estrategias e inferencias en la lectura crítica de textos. Memoria doctoral inédita. Madrid: Facultad de Educación, Departamento de Psicología Evolutiva y de la Educación-Universidad Complutense.

Marinac, J.; Ozanne, A. \& Woodyatt, G. (2000). Adult language input in the early childhood educational setting. Child Language Teaching and Therapy, 16(2), pp. 181-200.

McKeown, M.G. \& Beck, I.L. (2003). Taking advantage of readalouds to help children make sense of decontextualized language. In A. van Kleeck; A.A. Stahl \& E. B. Bauer (Eds.). On reading books to children: Parents and teachers, 6th edition (pp. 159-176). NJ: Mahwah, Erlbaum.

McKeown, M.G.; Beck, I.L. \& Blake, R.G.K. (2009). Rethinking reading comprehension instruction: A comparison for strategies and content approaches. Reading Research Quarterly, 44(3), pp. 218-253.

Mercer, N. (2000). Words and Minds: How We Use Language to Think Together. London: Routledge.

Neuman, S. \& Roskos, K. (2012). Helping children become more knowledgeable through text. The Reading Teacher, 66(3), pp. 207-210.

Nystrand, M. (1997). Opening dialogue: Understanding the dynamics of language and learning in the English classroom. New York: Teachers College Press.

Nystrand, M. \& Gamoran, A. (1991). Instructional discourse, student engagement, and literature achievement. Research in the Teaching of English, 25(3), pp. 261-290. 
Nystrand, M.; Wu, L.; Gamoran, A.; Zeiser, S. \& Long, D. (2003). Questions in time: Investigating the structure and dynamics of unfolding classroom discourse. Discourse Process, 5, pp. 135-196.

Paratore, J.; Cassano, CH. \& Schickedanz, J. (2011). Supporting Early (and Later) Literacy Development at Home and at School The long view. In M.L. Kamil; P.D. Pearson; E. Moje \& P. Afflerbach (Eds.). Handbook of Reading Research, IV (pp. 107-135). New York: Routledge.

Peterson, D.; Taylor, B.; Burmham, B. \& Schock, R. (2009). Reflective coaching conversations: A Missing Piece. The Reading Teacher, 62(6), pp. 500-509.

Peterson, D. \& Taylor, B. (2012). Using higher order questioning to accelerate students' growth in Reading. The Reading Teacher, 65(5), pp. 295-304.

Piaget, J. (1991). Seis estudios de psicología. Barcelona: Barral.

Santuiste, V. (2001). El pensamiento crítico en la práctica educativa. Madrid: Editorial Fugaz.

Snow, C.E. (1991). The theoretical basis for relationships between language and literacy in development. Journal of Research in Childhood Education, 6, pp. 5-10.

Swain, M. (2000). The output hypothesis and beyond: Mediating acquisition through collaborative dialogue. In J.P. Lantolf (Ed.). Sociocultural theory and second language learning (pp. 97-114). Oxford: Oxford Press.

Taylor, B.M.; Pearson, P.D.; Clark, K. \& Walpole, S. (1999). Effective schools and accomplished teachers: Lessons about primary grade reading instruction in low-income schools. Elementary School Journal, 101, pp. 121-165. 
Taylor, B.M.; Pearson, P.D.; Peterson, D.S. \& Rodriguez, M.C. (2003). Reading growth in high-poverty classrooms: The influence of teacher practices that encourage cognitive engagement in literacy learning. The Elementary School Journal, 104(1), pp. 3-28.

Torr, J. \& Clugston, L. (1999). A comparison between informational and narrative picture books as a context for reasoning between caregivers and 4-year-old children. Early Childhood Development and Care, 159, pp. 25-41.

Van Kleeck, A. (2003). Research on book sharing: Another critical look. In A. van Kleeck; A.A. Stahl \& E.B. Bauer (Eds.). On reading books to children: Parents and teachers (pp. 271-320). NJ: Mahwah/ Erlbaum.

Vygotsky, L.S. (1989). Pensamiento y lenguaje. Barcelona: Ediciones Fausto.

Wasik, B. (2010). What teachers can do to promote preschoolers' vocabulary development: Strategies from an effective language and literacy professional development coaching model. The Reading Teacher, 63(8), pp. 621-633.

Wasik, M.A. \& Bond, M.A. (2001). Beyond the pages of the book: Interactive book Reading and language development in preschool classrooms. Journal of Educational Psychology, 93, pp. 243-250.

Wegerif, R. \& Mercer, N. (2000). Language for Thinking. In H. Cowie; D. Aalsvoort \& N. Mercer. New Perspectives in Collaborative Learning. Oxford: Elsevier.

Wilkinson, I. \& Hye Son, E. (2011). A Dialogic Turn in Research on Learning and Teaching to Comprehend. In M.L. Kamil; P.D. Pearson, E. Moje \& P. Afflerbach (Eds.). Handbook of Reading Research, IV (pp. 107-135). New York: Routledge. 
Wittmer, D.S. \& Honing, A.S. (1991). Convergent or divergent? Teacher questions to three-year-old children in day care. Early Child Development and Care, 68, pp. 141-147.

Zucker, T.; Justice, L.M.; Piasta, S. \& Kaderaveck, J. (2010). Preschool teachers' literal and inferential questions and children's responses during whole-class shared Reading. Early Childhood Research Quarterly, 25, pp. 65-83.

Zuleta, O. (2005). La pedagogía de la pregunta, una contribución para el aprendizaje. Educare, 28, pp. 115-159. 